\title{
Clinical Immunogenicity of rHuPH20, a Hyaluronidase Enabling Subcutaneous Drug Administration
}

\author{
Sanna Rosengren, ${ }^{1,5}$ Samuel S. Dychter, ${ }^{1}$ Marie A. Printz, ${ }^{1}$ Lei Huang, ${ }^{1}$ Richard I. Schiff, ${ }^{2}$ Hans-Peter Schwarz, ${ }^{3}$ \\ John K. McVey, ${ }^{4}$ Fred H. Drake, ${ }^{1}$ Dan C. Maneval, ${ }^{1}$ Don A. Kennard, ${ }^{1}$ Gregory I. Frost, ${ }^{1}$ \\ Barry J. Sugarman, ${ }^{1}$ and Douglas B. Muchmore $^{1}$
}

Received 9 February 2015; accepted 1 May 2015; published online 13 May 2015

\begin{abstract}
Recombinant human PH20 hyaluronidase (rHuPH20) is used to facilitate dispersion of subcutaneously delivered fluids and drugs. This report summarizes rHuPH20 immunogenicity findings from clinical trials where rHuPH20 was co-administered with SC human immunoglobulin, trastuzumab, rituximab, or insulin. Plasma samples were obtained from evaluable subjects participating in ten different clinical trials as well as from healthy plasma donors. A bridging immunoassay and a modified hyaluronidase activity assay were used to determine rHuPH20-reactive antibody titers and neutralizing antibodies, respectively. rHuPH20-binding antibody populations from selected subjects with positive titers were affinity-purified and subjected to further characterization such as cross-reactivity with endogenous PH20. Among individual trials, the prevalence of pre-existing rHuPH20-reactive antibodies varied between 3 and 12\%, excepting the primary immunodeficiency (PID) studies. Incidence of treatment-induced rHuPH 20 antibodies was 2 to $18 \%$, with the highest titers $(81,920)$ observed in PID. No neutralizing antibodies were observed. Within most trials, the kinetics of antibody responses were comparable between pre-existing and treatment-induced antibody responses, although responses classified as persistent were more common in subjects with pre-existing titers. There was no association between antibody positivity and either local or systemic adverse events. Pre-existing and treatmentinduced antibody populations were of similar immunoglobulin isotypes and cross-reacted to endogenous PH20 to similar extents. No cross-reactivity to $\mathrm{PH} 20$ paralogs was detected. rHuPH20 induces only modest immunogenicity which has no association with adverse events. In addition, antibodies purified from baseline-positive individuals are qualitatively similar to those purified from individuals developing rHuPH20-reactive antibodies following exposure to the enzyme.
\end{abstract}

KEY WORDS: anti-drug antibodies; clinical trial; immunogenicity; rHuPH20; subcutaneous drug delivery.

\section{INTRODUCTION}

Hyaluronan (HA) is a mega-dalton glycosaminoglycan which constitutes a component of the extracellular matrix. Due to its viscosity and high water binding capacity, the HA viscoelastic gel forms a barrier to fluid flow and molecular movement through the interstitial collagenous matrix present in the hypodermis (1). This places a limit on the volume and composition of fluids and drugs that can be injected into the subcutaneous (SC) space. To overcome this limitation,

Electronic supplementary material The online version of this article (doi:10.1208/s12248-015-9782-0) contains supplementary material, which is available to authorized users.

\footnotetext{
${ }^{1}$ Halozyme Therapeutics, Inc., 11388 Sorrento Valley Road, San Diego, California 92121, USA.

${ }^{2}$ Baxter Bioscience, Deerfield, Illinois, USA.

${ }^{3}$ Baxter Bioscience, Vienna, Austria.

${ }^{4}$ Baxter Healthcare Corporation, Deerfield, Illinois, USA.

${ }^{5}$ To whom correspondence should be addressed. (e-mail: srosengren@halozyme.com)
}

hyaluronidases purified from bovine or ovine testes have been employed for decades to facilitate dispersion and absorption of subcutaneously injected solutions (2), but their clinical use has generally been limited by impurity profiles (1) and issues of hypersensitivity reactions (see for example (3)). More recently, the human testicular hyaluronidase ( $\mathrm{PH} 20$, encoded by the gene SPAM1) was cloned and described as a GPI-anchored protein (4) that possesses hyaluronidase activity (5). This discovery allowed the engineering of a recombinant form of the human $\mathrm{PH} 20$ protein (rHuPH20) lacking the GPI anchor domain (6) which can be purified to homogeneity with a specific activity 50 to 100 -fold greater than commercially available animalderived hyaluronidase products.

The suitability of using rHuPH20 to facilitate dispersion of injected fluids and drugs was demonstrated preclinically in models employing rodents (1) as well as pigs (7-9). Clinical trials with rHuPH20 have been undertaken in the areas of rapid large volume hydration $(10,11)$, rapid insulin and insulin analog delivery in subjects with diabetes (12-15), and delivery of large proteins such as immunoglobulins and monoclonal 
antibodies by SC rather than intravenous (IV) injections (9,16-21). Importantly, the use of SC rHuPH20 in combination with various drugs has been demonstrated to generate pharmacokinetic (PK) profiles that provide advantageous or comparable (i.e., noninferior) characteristics when compared to those obtained by IV injection of drug alone (16-20,22), and to increase the absorption rate of SC delivered insulin and its analogs $(12,14,15)$ as well as morphine $(23)$ among others. These data demonstrate the potential of rHuPH20 to facilitate the delivery of a variety of drugs in both acute and chronic clinical settings, either enabling transition to a more convenient, patient-friendly route of administration (e.g., conversion of treatments from IV to SC) or improving the PK profile of drugs that are typically administered SC (e.g., short acting insulin products).

Even though the use of human proteins as therapeutics can be expected to reduce immunogenicity relative to nonhuman proteins, the potential still exists of an antibody response. In fact, clinical immunogenicity of recombinant human proteins is frequently reported (24). The clinical relevance of those immune responses can vary widely and must be determined on a case-by-case basis through investigation of the impact of the immune response on product efficacy and safety. For example, anti-drug antibodies (ADA) may have significant impact on efficacy, through the development of neutralizing antibodies, and/ or drug PK (25). Cross-reactivity of neutralizing antibodies with an endogenous counterpart can also occur, and in some cases this has been associated with grave clinical consequences (26). The potential impact of ADA has led to the development of regulatory guidelines for immunogenicity testing and reporting $(27,28)$. Somewhat surprisingly, a number of cases have been reported in which preexisting antibodies to biotherapeutics are present in a certain percentage of the population, prior to any exposure to the drug in question $(29,30)$. The reason for these pre-existing antibodies is typically poorly understood, but their presence can complicate the interpretation of anti-drug antibody testing results obtained after administration of the drug in question.

Since $\mathrm{rHuPH} 20$ has been used clinically to facilitate dispersion and absorption of several co-administered biotherapeutic agents, it represents a unique opportunity to evaluate immunogenicity in multiple patient populations and dosing regimens. This report summarizes rHuPH20 immunogenicity findings from clinical trials where $\mathrm{rHuPH} 20$ was co-administered SC with human immunoglobulin, trastuzumab, rituximab, and insulin. In addition, a study was undertaken to determine the baseline prevalence of $\mathrm{rHuPH} 20$-reactive antibodies in the general population. Finally, antibodies from subjects who became positive for rHuPH20-reactive antibodies following exposure to $\mathrm{rHuPH} 20$ were characterized along with pre-existing antibodies from subjects who had never been treated with hyaluronidase. Results suggest that rHuPH20 induces only modest immunogenicity, with no meaningful changes to adverse event profiles. In addition, antibodies purified from individuals with pre-existing positive titers have isotypes and binding characteristics to endogenous PH20 similar to those purified from individuals developing rHuPH20-reactive antibodies following exposure to the protein.

\section{MATERIALS AND METHODS}

\section{Clinical Trials}

The designs of individual clinical trials for which immunogenicity analysis of $\mathrm{rHuPH} 20$ was conducted are described in detail in Supplemental 1. A summary of all included trials is provided in Table I.

\section{Sample Collection}

Blood samples, anticoagulated with EDTA, were obtained at the time of clinic visits according to the individual trial schedules. When relevant, samples were obtained immediately prior to the next scheduled treatment of $\mathrm{rHuPH} 20$ in order to avoid drug interference effects. Upon centrifugation, the resulting plasma was stored at approximately $-20^{\circ} \mathrm{C}$ and transported frozen to the testing laboratory.

\section{Assays for rHuPH20-Reactive and Neutralizing Antibodies}

In order to detect antibodies to rHuPH20, an electrochemiluminescence (ECL)-based bridging immunoassay was developed and validated according to recent regulatory guidelines $(27,28)$ and white papers $(31,32)$. After an overnight co-incubation of plasma sample diluted 1:5 with rHuPH20 conjugated to biotin and $\mathrm{rHuPH} 20$ conjugated to Sulfo-TAG (250 ng/mL each; Meso Scale Discovery, Rockville, MD), the resulting immune complex was captured onto streptavidin-coated plates and detected in a SECTOR 2400 instrument using ECL Read buffer (all Meso Scale Discovery). A three-tiered approach was employed consisting of screening, specificity testing using unconjugated rHuPH20 as a competitor, and two-fold step-wise titering in negative base pool plasma diluted 1:5. Statistically based cut points for screening positivity, specificity, and titration were established as recommended in (32). Individual sample titers were defined as the last dilution that yielded a positive response. The positive control for this method was a rabbit antirHuPH20 antibody affinity-purified from a pool of serum from three rabbits immunized with rHuPH20 in Freund's adjuvant. In order to determine assay sensitivity, a range of positive control concentrations was spiked into human plasma and evaluated using the bridging immunoassay to determine the threshold response versus the established screening cut point. While some of the ECL responses at $50 \mathrm{pg} / \mathrm{mL}$ fell above the assay cut point, all of the responses at $150 \mathrm{pg} / \mathrm{mL}$ were demonstrated to be greater than the cut point, and hence this conservative value was chosen to represent assay sensitivity. Taken into consideration the 1:5 dilution of plasma, the sensitivity of this assay was thus determined to be $\leq 750 \mathrm{pg} / \mathrm{mL}$, a threshold that was 660 -fold greater than the recommended $500 \mathrm{ng} / \mathrm{mL}$ for screening assays (28).

In the case of the HyQvia study, primary immunodeficiency (PID) subjects were treated with pools of human $\mathrm{IgG}$, which were shown to contain low levels of rHuPH20-reactive antibodies. This is a reflection of the baseline prevalence of anti-rHuPH20 in the general population described in "Results" section. Consequently, subjects in this study who were identified as not being able to produce mature antibodies due to their underlying immunodeficiency 
syndrome (X-linked agammaglobulinemia, severe combined immunodeficiency, or hyper IgM syndrome) nonetheless presented with $\mathrm{rHuPH} 20$-reactive antibody titers ranging from 10 to 80 , which were interpreted as the result of passive transfer of the antibodies contained in the therapeutic agent. Accordingly, HyQvia subjects were only considered to have a positive rHuPH20-reactive antibody response if a sample titer was $\geq 160$.

An assay for neutralizing antibodies (nAb) against rHuPH20 was based on the USP assay for hyaluronidase activity (33). Briefly, plasma samples diluted 1:20 were preincubated with $2 \mathrm{U} / \mathrm{mL}$ rHuPH 20 for at least an hour and was then allowed to digest high molecular weight hyaluronan for $30 \mathrm{~min}$. Addition of acidified serum resulted in turbidity at $640 \mathrm{~nm}$ due to the presence of precipitated hyaluronan which was monitored spectrophotometrically; any neutralizing antibody in the plasma sample diminished the capability of rHuPH20 activity to lower the turbidity. The minimum required plasma dilution of 1:20 was determined based on the potential for interference by known plasma components such as inter- $\alpha$-inhibitor (34).

For reporting immunogenicity responses, terms such as antibody prevalence, incidence, pre-existing and treatmentinduced antibodies, kinetics (transient vs. persistent), and titer increase over baseline (treatment-boosting) were defined as in (35). In addition, the requirement for an increase of two or more titering steps in order to consider a baseline-positive subject treatment-boosted was also defined as in (35). In this case, since the titering took place in 2-fold steps, that meant that a 4-fold or higher increase in titer was required for such classification.

\section{Adverse Events Analysis}

For trial 160603/902, each reported adverse event in subjects that developed rHuPH20-reactive antibodies was graded mild, moderate, or severe, and the number of adverse events occurring prior to and following the first positive titer were added for these subjects and expressed per time unit to yield an adverse events rate.

\section{rHuPH20-Reactive Antibody Purification}

For further characterization, rHuPH20-reactive antibodies were affinity-purified from 130 to $250 \mathrm{~mL}$ plasma from four HyQvia subjects who had previously yielded ECL bridging immunoassay titers in excess of 10,000 and who had titers of 2560 to 10,240 at the time of providing the sample used as a source for antibody. As controls, 700$800 \mathrm{~mL}$ obtained by plasmapheresis of four healthy volunteers with rHuPH20-reactive antibody titers of 160-640 was included. For affinity chromatography, Sepharose 4 Fast Flow resin (GE Healthcare, Pittsburgh, PA) was conjugated with rHuPH20 and packaged into Vantage chromatography columns (EMD Millipore, Billerica, MA) connected to an ÄKTA Purifier instrument (GE Life Sciences, Piscataway, NJ). Each plasma sample was diluted with 4 vol of TBS and loaded onto a fresh column, and antibodies were eluted with $0.1 \mathrm{M}$ glycine- $\mathrm{HCl}$ buffer, $\mathrm{pH} 2.5$, and immediately neutralized. Following dialysis against PBS, samples were concentrated to $0.5-1 \mathrm{~mL}$ using $30 \mathrm{kDa}$ molecular weight cut-off concentrators. 


\section{Antibody Isotyping}

The resulting purified antibody preparations were isotyped using a Human/Non-Human Primate isotyping kit (Meso Scale Discovery), and $\mathrm{IgG}$ subclasses were determined using a Human IgG subclass profile ELISA for IgG1-IgG3 (Life Technologies), and separately using a Human IgG4 ELISA (eBioscience). Within each preparation, the amount of each isotype/subclass was expressed as a percentage of the total amount of antibody.

\section{Evaluation of Antibody Cross-Reactivity}

Endogenous PH20 was released from human sperm (Fairfax Cryobank, Fairfax, VA) by PI-PLC (Sigma-Aldrich, St. Louis, MO) treatment after capacitation with calcium ionophore A23187 (Sigma-Aldrich). Recombinant human PH20 was also released by PI-PLC treatment from DG44CHO cells stably transfected with a full-length cDNA construct, and affinity-purified using Sepharose 4B conjugated to a rabbit polyclonal anti-rHuPH20 antibody and otherwise as above. The concentration of human $\mathrm{PH} 20$ in both preparations was determined using a sandwich ECL assay using the rabbit polyclonal antibody as a capture antibody in ECL High Bind plates (Meso Scale Discovery) and a Sulfo-TAG conjugate of the same antibody as a detecting antibody. Plates were read in a SECTOR 2400 instrument and concentrations determined using four-parameter logistic regression on ECL signal obtained using a dilution series of $\mathrm{rHuPH} 20$ as a standard.

In order to compare the binding of $\mathrm{rHuPH} 20$-reactive antibodies to rHuPH20 and endogenous full-length human $\mathrm{PH} 20$, plasma samples confirmed positive for rHuPH20reactive antibodies were diluted to yield an ECL signal around 5000 units (between 10- and 40-fold final dilution) and subjected to a version of the ECL bridging immunoassay described above wherein $\mathrm{rHuPH} 20$ conjugates were used at $100 \mathrm{ng} / \mathrm{mL}$. In these experiments, increasing concentrations of unconjugated rHuPH20, sperm-derived $\mathrm{PH} 20$, and $\mathrm{CHO}$-derived full-length PH20 were included to compete for binding to $\mathrm{rHuPH} 20$ reactive antibodies present in the sample. The resulting ECL signal/log competitor concentration curves were analyzed by four-parameter logistic regression where the top and bottom asymptotes were constrained as the ECL value obtained from the un-competed sample mean and the negative control mean, respectively, and the resulting IC50 value was reported.

To determine whether treatment-induced antibodies from subjects who participated in the HyQvia trial were capable of cross-reacting to recombinant human hyaluronidase (Hyal)1 and Hyal2, plasma samples from trial 160603/ 902 diluted 1:50 were assayed using the ECL bridging immunoassay described above in the presence of unlabeled test antigens added as competitors at a single concentration of $10 \mu \mathrm{g} / \mathrm{mL}$. Insulin glulisine and $\mathrm{rHuPH} 20$ were used as a negative and positive control competitors, respectively.

\section{RESULTS}

\section{Baseline Prevalence and Incidence of rHuPH20-Reactive Antibodies}

The baseline prevalence of $\mathrm{rHuPH} 20$-reactive antibodies prior to exposure to the recombinant protein is summarized for each clinical trial in Table II and varied between 3.3 and $12.1 \%$, except for the 160603/902 (primary immunodeficiency) trial where the baseline prevalence was only $1 / 87$. This single antibody-positive subject had previously been exposed to rHuPH20 in an earlier trial where immunogenicity was not monitored, and hence the provenance of this titer (pre-existing or treatment-induced) is not known. In a study of 961 healthy plasma donors between the ages of 18 and 65, the prevalence of

Table II. Prevalence and Incidence of rHuPH20-Binding Antibodies in All Clinical Trials

\begin{tabular}{|c|c|c|c|c|}
\hline Trial & Prevalence at baseline & $\begin{array}{l}\text { Treatment-induced } \\
\quad \text { (\# subjects) }\end{array}$ & $\begin{array}{l}\text { Treatment-boosted } \\
\quad \text { (\# subjects) }\end{array}$ & Total incidence \\
\hline $117-203$ & $2 / 46^{a}(4.3 \%)$ & 1 & 0 & $1 / 40(2.5 \%)$ \\
\hline $117-205$ & $13 / 117^{b}(11.1 \%)$ & 3 & 2 & $5 / 113(4.4 \%)$ \\
\hline $117-206$ & $4 / 120^{b}(3.3 \%)$ & 2 & 0 & $2 / 116(1.7 \%)$ \\
\hline $117-403$ & $38 / 456^{c}(8.3 \%)$ & 21 & 3 & $24 / 335(7.2 \%)$ \\
\hline HannaH & $22 / 290(7.6 \%)$ & 26 & 10 & $36 / 290(12.4 \%)$ \\
\hline SparkThera & $11 / 185$ (5.9\%) & 2 & 4 & $6 / 185(3.2 \%)$ \\
\hline SAWYER & $13 / 107^{d}(12.1 \%)$ & 3 & 3 & $6 / 96(6.3 \%)$ \\
\hline SABRINA & $28 / 257^{e}(10.9 \%)$ & 11 & 6 & $17 / 185(9.2 \%)$ \\
\hline All rHuPH20 trials except 160603/902 & $131 / 1578(8.3 \%)$ & 69 & 28 & $97 / 1360(7.1 \%)$ \\
\hline $160603 / 902$ & $1^{f} / 87^{b}(1.1 \%)$ & 14 & 1 & $15 / 83(18.1 \%)$ \\
\hline Normals & $56 / 961(5.8 \%)$ & $\mathrm{n} / \mathrm{a}$ & $\mathrm{n} / \mathrm{a}$ & $\mathrm{n} / \mathrm{a}$ \\
\hline
\end{tabular}

Definitions of prevalence, incidence, treatment-induced, and treatment-boosted as in (35). The italic text signifies the summation of all preceding lines

${ }^{a}$ Including 6 subjects where only a baseline sample was available

${ }^{b}$ Including 4 subjects where only a baseline sample was available

${ }^{c}$ Including 114 subjects randomized to receive standard continuous subcutaneous insulin infusion treatment, without rHuPH20, for the period studied; as well as 7 subjects randomized to receive a treatment regimen containing rHuPH20 but where only baseline and early termination samples were available

${ }^{d}$ Including 11 subjects where only a baseline sample was available

${ }^{e}$ Including 65 subjects randomized to the IV arm, as well as 7 subjects where only a baseline sample was available

${ }^{f}$ Note that this subject had previously participated in a trial of rHuPH20 and human IgG; however, during this previous trial immunogenicity monitoring was not performed. Hence, whether this subject was positive prior to any treatment with rHuPH20 is not known 
rHuPH20-reactive antibodies was $5.8 \%$, similar to those observed in the various disease populations. There was no significant difference in prevalence among men and women (34/450 vs. 22/455, Fisher's exact $p=0.13$ ), and no association between $\mathrm{rHuPH} 20$-reactive antibody positivity and age.

The incidence of rHuPH20-reactive antibodies following rHuPH20 exposure is also shown in Table II and is defined as the percentage of subjects that were negative at baseline but who became positive following exposure ("treatment-induced") plus the percentage of subjects that were positive at baseline and who had a 4-fold or higher increase of titer following exposure ("treatment-boosted"), as outlined in (35). In all cases, the number of treatment-induced subjects was greater than or similar to the number of treatment-boosted subjects. The overall incidence varied from 1.7 to $18.1 \%$.

Samples testing positive for $\mathrm{rHuPH} 20$-reactive antibodies were assayed for their ability to neutralize rHuPH20 enzyme activity. No instance of neutralizing antibodies was found in any sample from any of these clinical trials.

\section{Magnitude of rHuPH20-Reactive Antibody Responses}

Titers of rHuPH20-reactive antibodies in the individual clinical trials are shown in Fig. 1a. When all studies were combined (excluding the plasma donor survey study), the median maximum rHuPH20-reactive antibody titer in subjects with positive titers at baseline and subjects who became antibody-positive de novo following exposure to $\mathrm{rHuPH} 20$, respectively, was 40 (range 5-10,240) and 40 (range 5-81,920). Generally, maximum titers of baseline and treatment-induced antibodies within each population were of similar magnitude, with two exceptions: Trial 117-206 (type II diabetes, coadministered with insulin) where the maximum titer increased following exposure, but was still only at a median of 160, and trial 160603/902 (primary immunodeficiency, co-administered with human IgG) where the highest titers in all of the trials were observed in five subjects (Fig. 2).
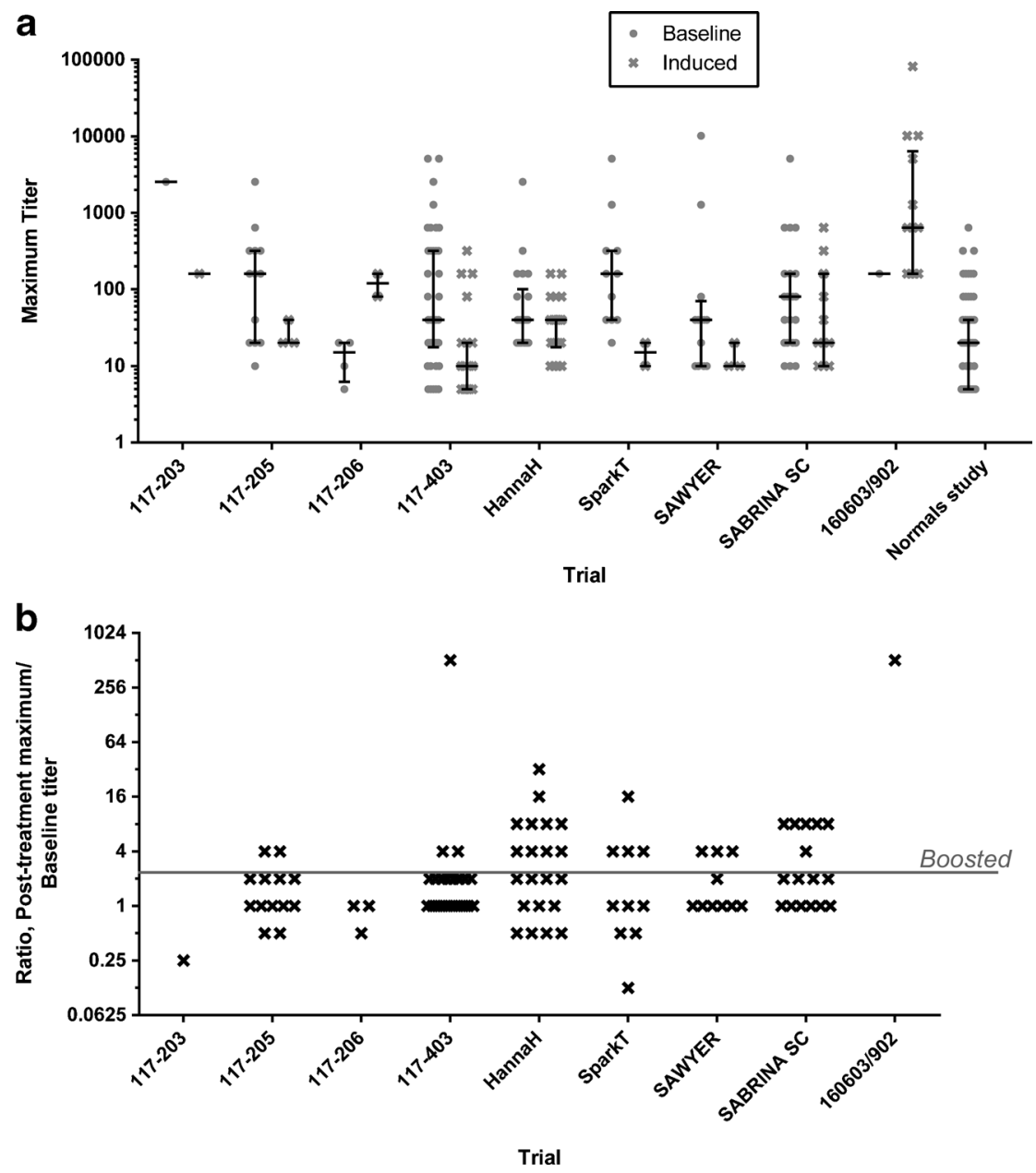

Fig. 1. a Maximum rHuPH20-reactive antibody titers observed prior to rHuPH20 exposure ("Baseline") and in subjects first testing positive following rHuPH20 exposure ("Induced"). Observations for individual subjects with median and interquartile range are indicated. b Maximum fold titer increase in baseline-positive subjects following rHuPH20 exposure. Individual subject observations are indicated. Subjects with more than a 2-fold titer increase following $\mathrm{rHuPH} 20$ exposure were considered treatment-boosted (threshold indicated by gray line). Ten baseline-positive subjects that were never positive following $\mathrm{rHuPH} 20$ exposure are not included in this figure 
a

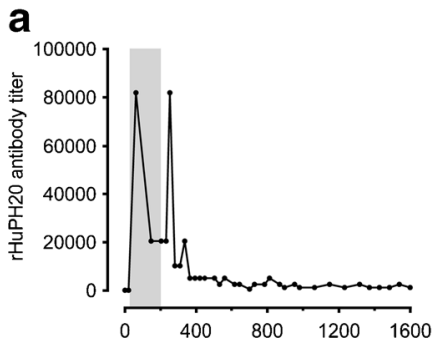

C

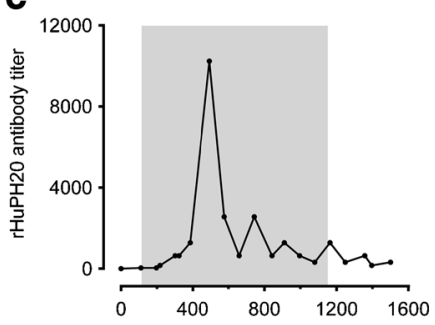

e

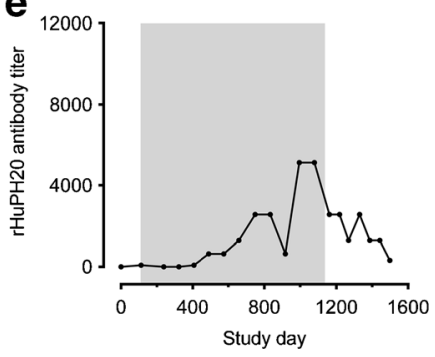

Fig. 2. Individual $\mathrm{rHuPH} 20$-binding antibody titers over time in the five subjects from trial 160603/902 who had maximum titers in excess of 5000. The shaded area in each panel indicates the period of rHuPH20 exposure. The titers of the single subject with a positive titer (of 160) prior to participating in this study are shown in panel a

In the baseline-positive subjects, the fold change following exposure (Fig. 1b) did not indicate systematic titer boosting by rHuPH20, but some subjects (enumerated in Table II and shown in Fig. $1 \mathrm{~b}$ above the gray threshold line) were considered treatment-boosted using the definition in (35). When all trials were considered together, treatmentboosting occurred in 29 of 110 evaluable baseline-positive subjects, whereas 71 baseline-positive subjects remained positive but not treatment-boosted, and ten baseline-positive subjects were not positive at any time following rHuPH20 exposure. The overall median fold titer change from baseline at post-exposure maximum was 2 (range 0.125-512). The highest extent of titer boosting was observed in one subject in trial 160603/902, who had previously been exposed to rHuPH20 (see above), as well as in one subject in trial 117403 who had a baseline titer at 5 and a titer at 12 months of 2560.

While the highest rHuPH20-binding antibody titer responses were observed in the 160603/902 trial, titers rapidly peaked and then decreased despite continued exposure to rHuPH20. Figure 2 depicts the longitudinal time versus titer profiles for five subjects over the course of the study who had maximum antibody titers in excess of 5000. The subject previously exposed to rHuPH20 (panel a) had a rapid antirHuPH20 antibody response, and rHuPH20 exposure in this subject was stopped out of an abundance of caution for further evaluation while antibody titers were monitored over time. All other subjects (panels b-e) completed the rHuPH20 treatment period. Typically, titers peaked after study day 450 and then reverted back down while the subjects continued to receive $\mathrm{rHuPH} 20$ treatments.

\section{Kinetics of rHuPH20-Reactive Antibody Responses}

Evaluations of the kinetics of antibody responses were possible using data sets from six clinical trials where the period of antibody monitoring exceeded 16 weeks, or approximately 5 half-lives of $\mathrm{IgG}$, in at least some of the subjects in the trial (35). The timing of the onset of antibody positivity (in subjects negative at baseline) and of the first sign of treatment boosting (in subjects positive at baseline) is summarized in Fig. 3a and is given as study day without taking the overall duration of the study into account, nor the frequency of rHuPH20 administration, so comparison between trials is not particularly informative. However, within trials, the onset of de novo antibody positivity and of antibody boosting was comparable, with one exception: the single baseline-positive subject in 160603/902 (who was previously exposed to rHuPH20, see above) had a very rapid onset of titer boosting compared to the timing of antibody positivity onset in the baseline-negative subjects in this trial. The timing of antibody titer maxima (Fig. 3b) also displayed a comparable pattern for baseline-positive subjects and for subjects with treatment-induced antibodies within each trial, except for trial 160603/902.

Persistence of antibody positivity was determined in all subjects where the period of antibody monitoring exceeded 16 weeks. As seen in Fig. 4a, the duration of antibody positivity was generally longer in subjects who tested positive for antibodies before rHuPH20 exposure, except in the SABRINA trial where considerable overlap was observed. When the definitions for "persistent" antibody responses were applied according to (35) (see Fig. 4 legend for definition), the percent of antibody-positive subjects having persistent antibodies was always higher in the baselinepositive population (75 to $100 \%)$ than in the treatmentinduced population ( 0 to $25 \%$ ) (Fig. $4 \mathrm{~b}$ ).

\section{Lack of Association Between rHuPH20-Reactive Antibodies and Adverse Events}

In order to determine whether any association between the conversion to antibody positivity and adverse events could be detected, data from trial 160603/902 were analyzed for all subjects who developed antibodies following rHuPH20 exposure (Table III). Results indicate that time-adjusted rates of both local and systemic adverse events classified as mild, moderate, and severe were comparable prior to and following first positive titer, with a slight decrease in each adverse event rate following $\mathrm{rHuPH} 20$-antibody positivity. The similarity of adverse event rates pre- and post-rHuPH20 antibody positivity also held true when the 15 subjects from trial 160603/902 were analyzed as a subset, or when the five subjects with the highest titers from this trial were considered on their own. 

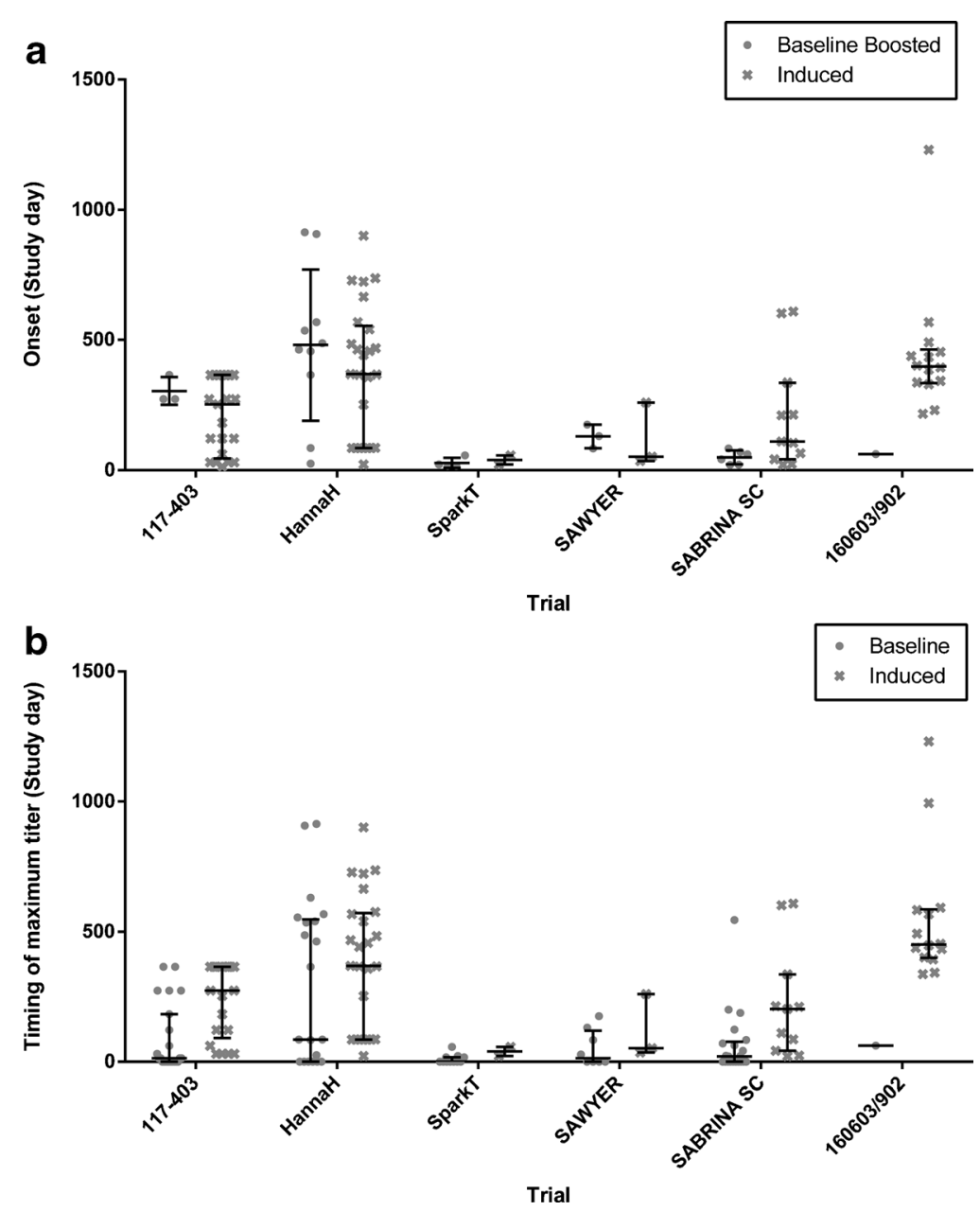

Fig. 3. a Onset of rHuPH20-reactive antibody boosting (see definition in legend for Fig. 1) in subjects testing positive for antibodies prior to treatment with $\mathrm{rHuPH} 20$ ("Baseline Boosted") and in subjects first testing positive following rHuPH20 exposure ("Induced"). b Timing of maximum rHuPH20-reactive antibody titers in subjects testing positive for antibodies prior to treatment with $\mathrm{rHuPH} 20$ ("Baseline") and subjects first testing positive following rHuPH20 exposure ("Induced"). Individual observations with median and interquartile range are indicated

\section{Characterization of rHuPH20-Reactive Antibodies}

In order to compare the characteristics of $\mathrm{rHuPH} 20$ reactive antibodies that emerged following treatment with rHuPH20 (trial 160603/902, panels a-c and e in Fig. 2) and those present in baseline-positive individuals, rHuPH20 affinity chromatography was used to purify antibody preparations from subjects with titers at the time of plasma donation shown in Fig. 5a. Following purification, the lowest antibody concentration that could be detected using the bridging immunoassay was highly variable but within similar ranges for the two populations (Fig. 5b). Isotyping demonstrated that the two types of antibody preparations contained comparable proportions of $\operatorname{IgM}, \operatorname{IgG}$, and $\operatorname{IgA}$ (Fig. 5c), and percentages of individual IgG subclasses were also similar with the exception of a single elevated $\operatorname{IgG} 4$ level in the treatment-induced group (Fig. 5d).

Cross-reactivity of rHuPH20-reactive antibodies to related endogenous proteins, i.e., human sperm-derived PH20 and paralogous human hyaluronidase proteins, was studied because of the shared sequence homology between rHuPH20 and these other hyaluronidases $(100 \%$ between rHuPH 20 and endogenous $\mathrm{PH} 20$ with the exception of the C-terminal truncation, and between 34 and $42 \%$ homology with hyaluronidase (Hyal)-1, $-2,-3$, and -4 (36)). This evaluation was performed using a modification of the bridging immunoassay, where unlabeled proteins were used to compete in the bridging reaction. The data indicate that endogenous $\mathrm{PH} 20$ yielded similar results whether treatment-induced and pre-existing antibody preparations were tested (Fig. 6a), whereas rHuPH20 tended to inhibit bridging reactions with treatment-induced antibody preparations more efficiently than those with preexisting antibodies (Fig. 6b). Under these circumstances, the highest concentration of rHuPH 20 tested $(2 \mu \mathrm{g} / \mathrm{mL})$ inhibited the bridging signal observed with both treatment-induced and pre-existing antibodies by $>99 \%$.

In separate experiments, plasma samples from four $160603 / 902$ subjects with the highest titer of rHuPH20binding antibodies (as shown in Fig. 2a-d), as well as two subjects deemed antibody-negative, were evaluated for crossreactivity to other human hyaluronidases (Hyal-1 and Hyal-2) 

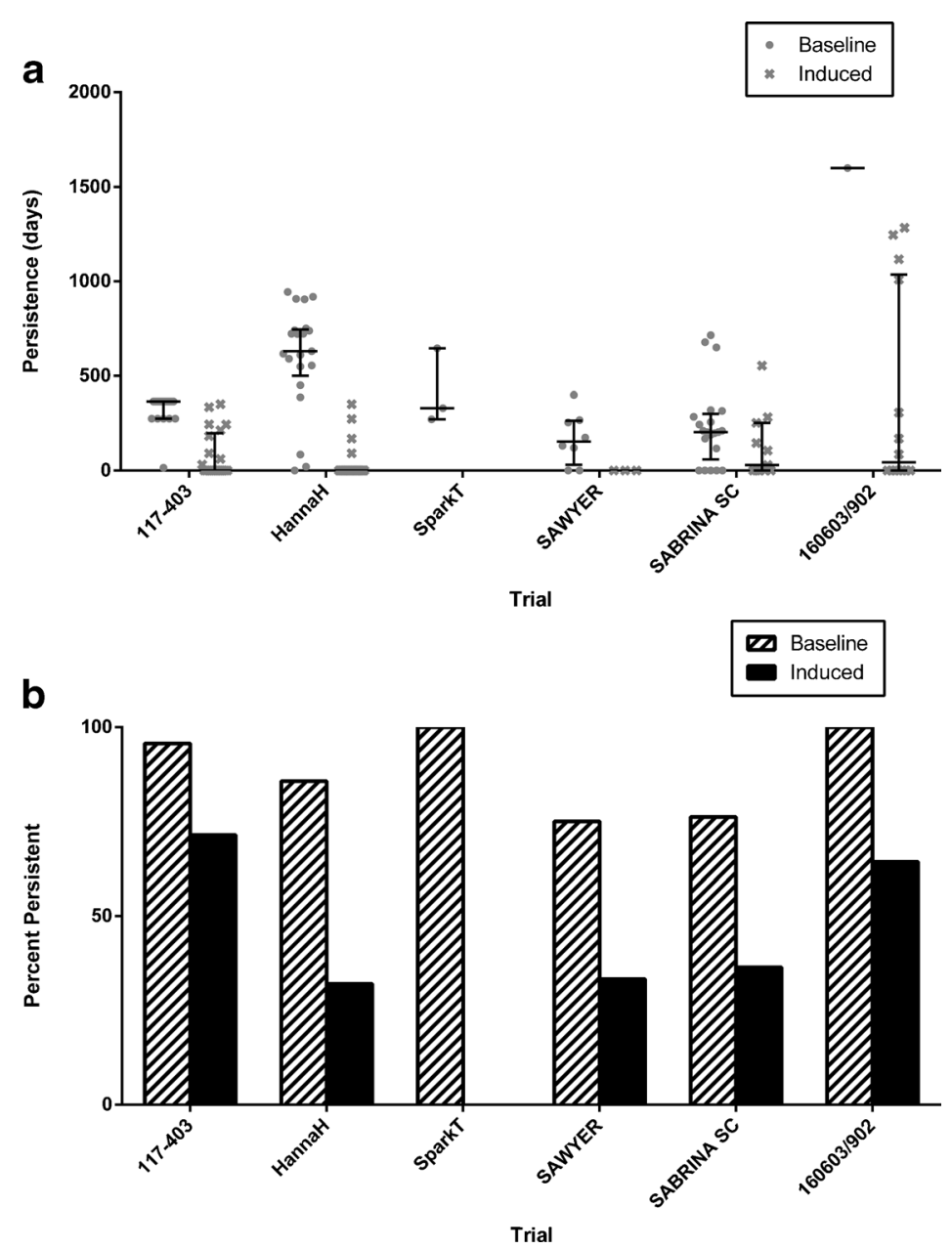

Fig. 4. Persistence of rHuPH20-reactive antibodies in subjects testing positive for antibodies prior to treatment with $\mathrm{rHuPH} 20$ ("Baseline") and subjects first testing positive following rHuPH20 exposure ("Induced"). a Individual observations with median and interquartile range are indicated. b Percentage of subjects with "Persistent" antibodies according to the definition in (35) (first and last antibodypositive samples, irrespective of any negative samples in between, are separated by a period of 16 weeks or longer, or first antibody-positive sample is obtained less than 16 weeks before last sample obtained or end of study)

Table III. Summary of Adverse Events ${ }^{a}$ in Baxter Trials in Subjects Who Developed Anti-rHuPH20 Antibodies

\begin{tabular}{|c|c|c|c|c|c|}
\hline \multirow[b]{2}{*}{ Adverse events data set } & \multirow[b]{2}{*}{ Severity } & \multicolumn{2}{|c|}{ Before first positive anti-rHuPH20 titer } & \multicolumn{2}{|c|}{ After first positive anti-rHuPH20 titer } \\
\hline & & Number of events & Rate $^{b}$ & Number of events & Rate \\
\hline \multirow[t]{4}{*}{ Total adverse events } & Mild & 72 & 7.14 & 202 & 8.73 \\
\hline & Moderate & 56 & 5.55 & 55 & 2.38 \\
\hline & Severe & 10 & 0.99 & 9 & 0.39 \\
\hline & Total & 138 & 13.69 & 266 & 11.50 \\
\hline \multirow[t]{4}{*}{ Systemic adverse events } & Mild & 49 & 4.86 & 135 & 5.84 \\
\hline & Moderate & 35 & 3.47 & 51 & 2.20 \\
\hline & Severe & 7 & 0.69 & 9 & 0.39 \\
\hline & Total & 91 & 9.02 & 195 & 8.43 \\
\hline \multirow[t]{4}{*}{ Local adverse events } & Mild & 23 & 2.28 & 67 & 2.90 \\
\hline & Moderate & 21 & 2.08 & 4 & 0.17 \\
\hline & Severe & 3 & 0.30 & 0 & 0.00 \\
\hline & Total & 47 & 4.66 & 71 & 3.07 \\
\hline
\end{tabular}

${ }^{a}$ Adverse events excluding infections

${ }^{b}$ Rate =number of adverse events divided by number of years in the respective observation period, which includes 3683 and 8449 total cumulative subject days (10.08 and 23.13 total cumulative subject years) of observation prior to and following the first exposure to rHuPH20, respectively 
a

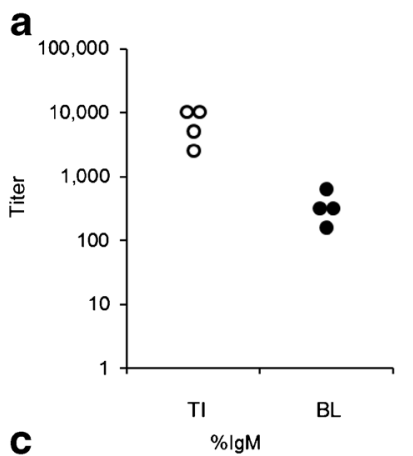

C
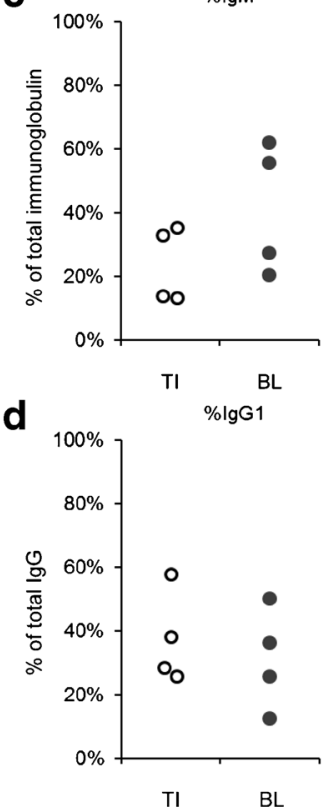

b
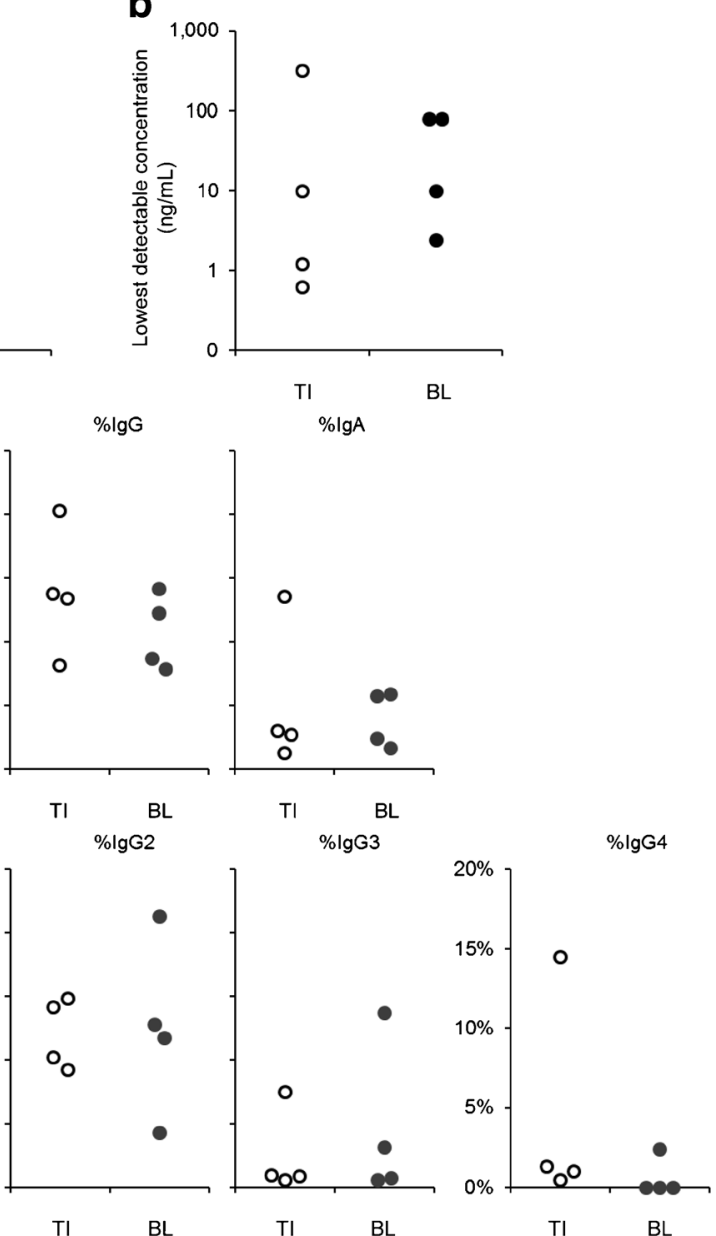

Fig. 5. Purification and characterization of rHuPH20-reactive antibodies from PID subjects with de novo positive antibody titers following exposure to $\mathrm{rHuPH} 20$ (treatment-induced, "TI") as well as from the baseline-positive population with preexisting rHuPH20 antibodies (baseline, "BL"). a Plasma titers at the time of antibody purification. b Lowest detectable concentration of purified rHuPH20-reactive antibodies in the ECL bridging assay. c Isotypes. d IgG subclasses. Observations from individual antibody preparations from four individuals each are indicated

as determined by competition by unlabeled protein at $10 \mu \mathrm{g} / \mathrm{mL}$ in the bridging immunoassay. In these studies, unlabeled $\mathrm{rHuPH} 20$ inhibited the bridging reaction between the biotinylated- and ruthenylated-rHuPH20 by greater than $99 \%$ in all samples except for the two negative samples $(<23 \%)$. In contrast, the percentage inhibition for recombinant Hyal-1 and Hyal-2 as well as the glulisine negative control was less than $23 \%$ for all samples.

\section{DISCUSSION}

The recombinant hyaluronidase, $\mathrm{rHuPH} 20$, is used to facilitate SC delivery of protein therapeutics that would normally require intravenous infusion and can also be used to intentionally improve the PK profile of drugs that are usually administered by SC route. It is typically co-infused SC along with the therapeutic, either using sequential administration or using co-formulated drug product. In either case, rHuPH20 allows the therapeutic agent to permeate more readily through the $\mathrm{SC}$ space and gain access to the central circulation via either the capillaries, for small molecule therapeutics, or the lymphatics, for large molecule therapeutics. This report summarizes the clinical immunogenicity responses to $\mathrm{rHuPH} 20$ in several clinical trials, including rHuPH20 administered in combination with insulin, therapeutic antibodies, or human $\mathrm{IgG}$ to a total of 1526 subjects from diverse populations. Overall, exposure to $\mathrm{rHuPH} 20$ was associated with emergence of $\mathrm{rHuPH} 20$-reactive antibodies in 3 to $18 \%$ of treated individuals, depending on the trial. Of the 83 individuals who were antibody-negative prior to treatment and developed rHuPH20-binding titers after exposure to the protein, only three experienced maximum titers in excess of 10,000 using a highly sensitive bridging immunoassay. In addition, 28 subjects with pre-existing rHuPH20-reactive antibodies prior to exposure experienced treatment boosting, as defined by an increase in titer of two titering steps or more following treatment, and one of these experienced a maximum titer of 81,920 , the highest titer observed following rHuPH20 exposure in this data set. Importantly, no neutralizing antibodies were identified in any of these trials. 


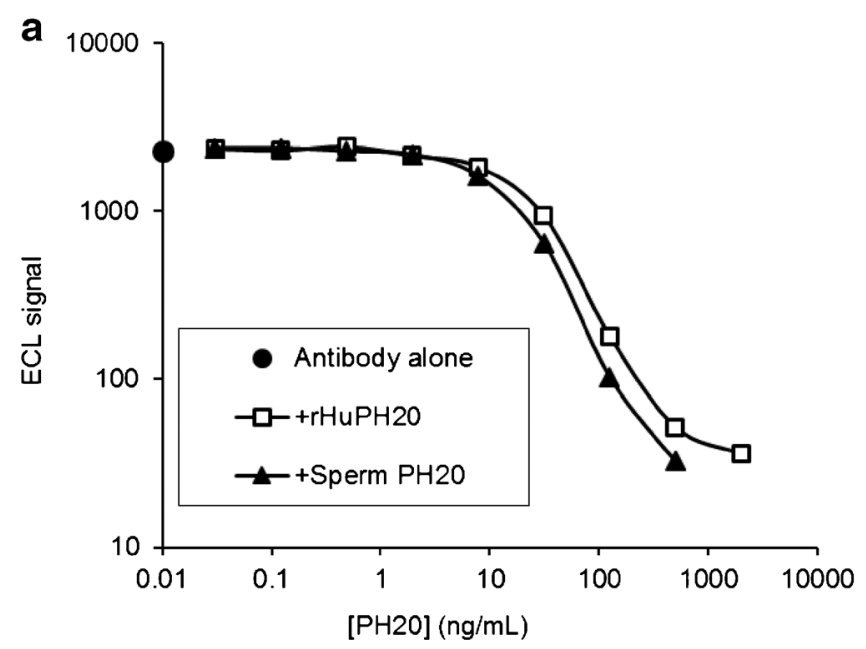

b

Sperm $\mathrm{PH} 2 \mathrm{O}$

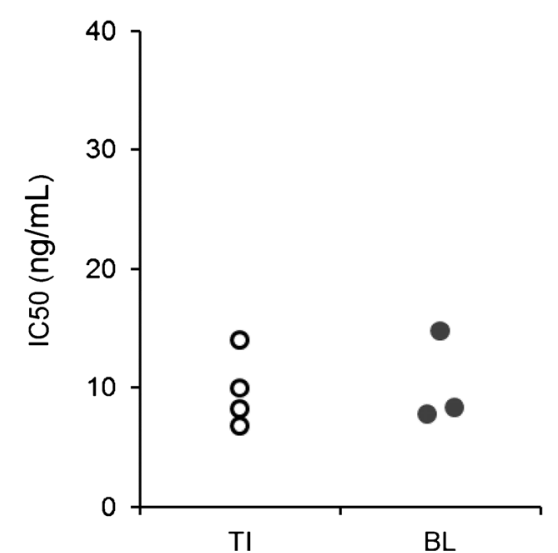

$\mathrm{rHuPH} 20$

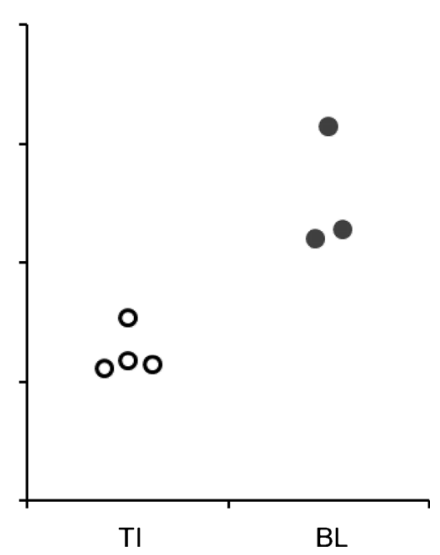

Fig. 6. Antibody cross-reactivity to endogenous $\mathrm{PH} 20$. a Representative example of results obtained in the ECL bridging competition assay with an individual $\mathrm{rHuPH} 20$ reactive antibody preparation when increasing concentrations of $\mathrm{rHuPH} 20$ or $\mathrm{PH} 20$ isolated from human sperm were included. b Resulting IC50 values calculated from individual antibody preparations. Open circles, rHuPH20-reactive antibodies from PID subjects with de novo positive antibody titers following exposure to rHuPH20. Closed circles, rHuPH20-reactive antibodies from the baseline-positive population

The clinical importance of ADA responses can manifest itself in various ways: neutralizing antibodies can alter drug efficacy (25) and, in the worst case, can cross-neutralize the endogenous counterpart (26); both binding and neutralizing antibodies can have an impact of the PK of the drug (25); and circulating ADA-drug immune complexes can form with potential deleterious safety consequences (37). In the case of rHuPH20, no potential impact on function of endogenous PH20 should be expected since no neutralizing antibodies were observed. No clinical data are yet available regarding the potential effect of anti-rHuPH20 antibodies on $\mathrm{PK}$ of rHuPH20 itself; however, the action of rHuPH20 is localized to the SC space and no evidence of systemic exposure can be detected at the doses used (17). This might be related to the rapid elimination of $\mathrm{rHuPH} 20$ from the central compartment. Even when infused IV in human volunteers, rHuPH20 has a terminal elimination half-life of only 5-6 min (manuscript in preparation). Finally, with regards to immune complex formation, whereas $\mathrm{IgG}$ can theoretically distribute to the skin and lymph (38), there was no difference in adverse event rates in subjects who eventually became positive for $\mathrm{rHuPH} 20$-binding antibodies when periods prior to and following first positive titer were compared.

A consistent finding through all the trials was the presence of rHuPH20-binding antibodies in a small percentage of subjects prior to $\mathrm{rHuPH} 20$ exposure. This was confirmed in a separate survey study of 961 healthy plasma donors where the prevalence of pre-existing antibodies was determined to be $6 \%$. The basis for why they are sometimes present remains unknown but may be related to immune reactivity to endogenous PH20, a GPIanchored hyaluronidase normally present on the apical head of male sperm $(4,5)$. Whatever their provenance, the presence of pre-existing rHuPH20-reactive antibodies does not appear to be associated with systematic titer boosting after exposure to rHuPH20 in the majority of subjects. Importantly, there were no adverse events associated with anti-rHuPH20 reactivity, with or without boosting.

The comparison of several clinical trials with unique study designs, dose levels of rHuPH20, and sampling schedules can be fraught with difficulty, which can limit the conclusions that can be 
drawn. In the current case, the various trials yielded similar results in terms of the nature of the $\mathrm{rHuPH} 20$ immunogenicity response: First, within trials, pre-existing and treatment-induced antibody titers were similar, as were their kinetics of onset of positivity versus onset of boosting as well as time to maximum titer. Second, if treatment-induced $\mathrm{rHuPH} 20$ antibody responses occurred, they tended to be more transient in nature than for pre-existing antibodies. The reason for this is currently unknown, but could possibly be related to the existence of a robust and long-lasting immune response to $\mathrm{PH} 20$ in individuals with preexisting antibodies which predisposed towards more durable boosting. No data exists to date to address this question. Third, no neutralizing antibodies were observed in any clinical study.

The notable exception to this pattern was the response observed in the 160603/902 trial, where rHuPH20 was administered in combination with human $\mathrm{IgG}$ in subjects with primary immunodeficiency and induced a durable rHuPH20-binding antibody response with titers in excess of 5000 in five subjects. These titers generally diminished despite continuing treatment, and no neutralizing antibodies were ever observed; however, the nature of these responses was different from those observed in other clinical trials with rHuPH20. The reason for this could possibly be sought in the design of the trials (e.g., dosing frequency, total dose) or the co-administered therapeutic, in this case human $\mathrm{IgG}$ purified and pooled from plasma donors, which could contain low levels of rHuPH20-reactive antibodies due to the prevalence of such antibodies in the general population as described above. This latter notion was confirmed by analysis of preparations of human $\mathrm{IgG}$ which contained approximately $0.01-0.02 \%$ of $\mathrm{rHuPH} 20$-reactive antibodies (unpublished observations). Besides these differences, another potential reason for the differential immunogenicity response to rHuPH20 in the 160603/902 trial was the subject population. Primary immunodeficiencies constitute a diverse group of disorders of monogenic or polygenic origin, where the genetic basis of the disorder in the more frequently diagnosed disorders such as CVID is frequently unknown (39). Many of these diseases predispose affected subjects to dysregulated T-cell tolerance and autoimmunity $(40,41)$, including CVID (42), which was diagnosed in three of the five cases in which the highest $\mathrm{rHuPH} 20$-reactive antibody titers were observed.

Beside the possibility that antibodies to rHuPH20 could attenuate in vivo enzymatic activity and produce a loss of dispersive effect at the injection site, the other theoretical concern is whether these antibodies could affect the functionality of endogenous $\mathrm{PH} 20$ or closely related enzymes. In vitro binding of anti-rHuPH20 antibodies to endogenous $\mathrm{PH} 20$ would be expected because of the high homology between $\mathrm{rHuPH} 20$ and $\mathrm{PH} 20$. On the other hand, binding of such antibodies to $\mathrm{PH} 20$ in vivo would be limited by the limited tissue expression profile of PH20 (adult male reproductive tract) as well as the blood-testis and blood-epididymal barriers limiting access of antibodies in systemic circulation to that milieu (43).

In order to address some of these concerns, treatmentinduced $\mathrm{rHuPH} 20$-reactive antibodies as well as those from baseline-positive subjects were affinity-purified using rHuPH20coupled resin and extensively characterized using various methods such as antibody titer, antibody isotype, and in vitro cross-reactivity to endogenous human $\mathrm{PH} 20$ and related hyaluronidases. The resulting antibody preparations from rHuPH20-treated and baseline-positive subjects had similar isotypes and $\mathrm{IgG}$ subclasses, suggesting that the degree to which immune class switching had taken place was comparable in both populations. Cross-reactivity analysis demonstrated that both types of antibody preparations bound to endogenous PH20 in vitro to a similar degree, whereas the treatment-induced antibodies bound somewhat better to rHuPH20 than did the pre-existing ones. This indicates that rHuPH20-binding antibodies emerging following treatment, even in the case of the comparably high titers occasionally observed in the 160603/902 trial, share molecular and immunological characteristics with those observed in more than $5 \%$ in the general population.

With respect to other human hyaluronidases, the risk of anti-rHuPH20 antibodies binding to them is lower because the primary structure of $\mathrm{rHuPH} 20$ is only distally related (i.e., 34 to $42 \%$ homology) to Hyal-1, $-2,-3$, and -4 (36). In support of this notion, none of these plasma samples cross-reacted to recombinant Hyal-1 or Hyal-2 when using the modified bridging immunoassay. This conclusion is corroborated by results from a tissue cross-reactivity study using the affinity-purified antirHuPH20 antibodies to stain different human tissues (unpublished observations); the only specific immunohistochemical staining observed was in the seminiferous tubules in the testis. This expression profile differs from that observed for other hyaluronidases, which are expressed in many tissues including lung, liver, skeletal muscle, and kidney (44). If anti-rHuPH20 antibodies cross-reacted with other hyaluronidase family members, multiple tissues would have been expected to be positive in the tissue cross-reactivity study. Thus, the lack of signal in multiple assay systems indicates that anti-rHuPH20 antibodies did not cross-react with $\mathrm{PH} 20$ paralogs.

These observations serve to alleviate potential concerns raised by the apparent binding (although not neutralization) of a treatment-induced antibody to an endogenous protein involved in aspects of reproduction and are further supported by published reports in which several attempts were made to immunize males with $\mathrm{PH} 20$ as an immunocontraceptive approach in animal models. These studies involved rabbits $(45,46)$, mice (47), and guinea pigs (48), and only the latter experienced infertility following $\mathrm{PH} 20$ immunization with a crude testicular extract that resulted in autoimmune orchitis (49). Furthermore, sperm from mice lacking PH20 were able to fertilize eggs, albeit in a somewhat delayed manner (50).

In conclusion, $\mathrm{rHuPH} 20$ is a recombinant human protein that appears to have modest immunogenicity and no deleterious effects on efficacy or adverse events. In addition, antibodies that bind to $\mathrm{rHuPH} 20$ are present in about one in 20 healthy individuals not exposed to the protein, and the functional characteristics of preexisting and treatment-induced antibodies are similar. Therefore, rHuPH20 continues to constitute an attractive therapeutic option for delivering large molecules and fluid volumes via the SC route as an alternative to IV administration.

\section{ACKNOWLEDGMENTS}

The authors wish to thank Drs. Robert Connor and Ge Wei for assistance with protein purifications, and Dr. Rose Sekulovich for assistance with extended characterization of purified antibodies. These studies were funded by Halozyme Therapeutics, Baxter Healthcare Corporation, and Roche. 
Open Access This article is distributed under the terms of the Creative Commons Attribution 4.0 International License (http://creativecommons.org/licenses/by/4.0/), which permits unrestricted use, distribution, and reproduction in any medium, provided you give appropriate credit to the original author(s) and the source, provide a link to the Creative Commons license, and indicate if changes were made.

\section{REFERENCES}

1. Bookbinder LH, Hofer A, Haller MF, Zepeda ML, Keller GA, Lim JE, et al. A recombinant human enzyme for enhanced interstitial transport of therapeutics. J Control Release. 2006;114(2):230-41.

2. Menzel EJ, Farr C. Hyaluronidase and its substrate hyaluronan: biochemistry, biological activities and therapeutic uses. Cancer Lett. 1998;131(1):3-11.

3. Kempeneers A, Dralands L, Ceuppens J. Hyaluronidase induced orbital pseudotumor as complication of retrobulbar anesthesia. Bull Soc Belge Ophtalmol. 1992;243:159-66.

4. Lin Y, Kimmel LH, Myles DG, Primakoff P. Molecular cloning of the human and monkey sperm surface protein PH-20. Proc Natl Acad Sci U S A. 1993;90(21):10071-5.

5. Gmachl M, Sagan S, Ketter S, Kreil G. The human sperm protein $\mathrm{PH}-20$ has hyaluronidase activity. FEBS Lett. 1993;336(3):545-8.

6. Frost GI. Recombinant human hyaluronidase (rHuPH20): an enabling platform for subcutaneous drug and fluid administration. Expert Opin Drug Deliv. 2007;4(4):427-40.

7. Kang D, Jadin L, Nekoroski T, Drake F, Zepeda M. Recombinant human hyaluronidase $\mathrm{PH} 20$ (rHuPH20) facilitates subcutaneous infusions of large volumes of immunoglobulin in a swine model. Drug Deliv Transl Res. 2012;2(4):254-64.

8. Kang DW, Oh DA, Fu GY, Anderson JM, Zepeda ML. Porcine model to evaluate local tissue tolerability associated with subcutaneous delivery of protein. J Pharmacol Toxicol Methods. 2013;67(3):140-7.

9. Dychter SS, Harrigan R, Bahn JD, Printz MA, Sugarman BJ, DeNoia E, et al. Tolerability and pharmacokinetic properties of ondansetron administered subcutaneously with recombinant human hyaluronidase in minipigs and healthy volunteers. Clin Ther. 2014;36(2):211-24.

10. Thomas JR, Yocum RC, Haller MF, von Gunten CF. Assessing the role of human recombinant hyaluronidase in gravity-driven subcutaneous hydration: the INFUSE-LR study. J Palliat Med. 2007;10(6):1312-20.

11. Allen CH, Etzwiler LS, Miller MK, Maher G, Mace S, Hostetler MA, et al. Recombinant human hyaluronidase-enabled subcutaneous pediatric rehydration. Pediatrics. 2009;124(5):e858-67.

12. Hompesch M, Muchmore DB, Morrow L, Vaughn DE. Accelerated insulin pharmacokinetics and improved postprandial glycemic control in patients with type 1 diabetes after coadministration of prandial insulins with hyaluronidase. Diabetes Care. 2011;34(3):666-8.

13. Vaughn DE, Muchmore DB. Use of recombinant human hyaluronidase to accelerate rapid insulin analogue absorption: experience with subcutaneous injection and continuous infusion. Endocr Pract. 2011;17(6):914-21.

14. Hompesch $\mathrm{M}$, Muchmore DB, Morrow L, Ludington $\mathrm{E}$, Vaughn DE. Improved postprandial glycemic control in patients with type 2 diabetes from subcutaneous injection of insulin lispro with hyaluronidase. Diabetes Technol Ther. 2012;14(3):218-24.

15. Morrow L, Muchmore DB, Hompesch M, Ludington EA, Vaughn DE. Comparative pharmacokinetics and insulin action for three rapid-acting insulin analogs injected subcutaneously with and without hyaluronidase. Diabetes Care. 2012;36(2):273-5.

16. Wasserman RL. Progress in gammaglobulin therapy for immunodeficiency: from subcutaneous to intravenous infusions and back again. J Clin Immunol. 2012;32(6):1153-64.
17. Wynne C, Harvey V, Schwabe C, Waaka D, McIntyre C, Bittner B. Comparison of subcutaneous and intravenous administration of trastuzumab: a phase $\mathrm{I} / \mathrm{Ib}$ trial in healthy male volunteers and patients with HER2-positive breast cancer. J Clin Pharmacol. 2013;53(2):192-201.

18. Ismael G, Hegg R, Muehlbauer S, Heinzmann D, Lum B, Kim $\mathrm{SB}$, et al. Subcutaneous versus intravenous administration of (neo)adjuvant trastuzumab in patients with HER2-positive, clinical stage I-III breast cancer (HannaH study): a phase 3, open-label, multicentre, randomised trial. Lancet Oncol. 2012;13(9):869-78.

19. Morcos PN, Zhang X, McIntyre C, Bittner B, Rowell L, Hussain Z. Pharmacokinetics and pharmacodynamics of single subcutaneous doses of tocilizumab administered with or without rHuPH20. Int J Clin Pharmacol Ther. 2013;51(7):537-48.

20. Shpilberg O, Jackisch C. Subcutaneous administration of rituximab (MabThera) and trastuzumab (Herceptin) using hyaluronidase. Br J Cancer. 2013;109(6):1556-61.

21. Jackisch C, Kim SB, Semiglazov V, Melichar B, Pivot X, Hillenbach $\mathrm{C}$, et al. Subcutaneous versus intravenous formulation of trastuzumab for HER2-positive early breast cancer: updated results from the phase III HannaH study. Ann Oncol. 2014

22. Harb G, Lebel F, Battikha J, Thackara JW. Safety and pharmacokinetics of subcutaneous ceftriaxone administered with or without recombinant human hyaluronidase $(\mathrm{rHuPH} 20)$ versus intravenous ceftriaxone administration in adult volunteers. Curr Med Res Opin. 2010;26(2):279-88.

23. Thomas JR, Wallace MS, Yocum RC, Vaughn DE, Haller MF, Flament J. The INFUSE-Morphine study: use of recombinant human hyaluronidase (rHuPH20) to enhance the absorption of subcutaneously administered morphine in patients with advanced illness. J Pain Symptom Manag. 2009;38(5):663-72.

24. Baker MP, Reynolds HM, Lumicisi B, Bryson CJ. Immunogenicity of protein therapeutics: the key causes, consequences and challenges. Self Nonself. 2010;1(4):314-22.

25. Chirmule N, Jawa V, Meibohm B. Immunogenicity to therapeutic proteins: impact on PK/PD and efficacy. AAPS J. 2012;14(2):296-302.

26. Bennett CL, Luminari S, Nissenson AR, Tallman MS, Klinge SA, McWilliams $\mathrm{N}$, et al. Pure red-cell aplasia and epoetin therapy. N Engl J Med. 2004;351(14):1403-8.

27. EMA. Guideline on immunogenicity assessment of biotechnologyderived therapeutic proteins. European Medicines Agency; 2007. p. $1-18$.

28. FDA. Draft guidance for industry: assay development for immunogenicity testing of therapeutic proteins. US Department of Health and Human Services 2009, p. 1-24.

29. Xue L, Fiscella M, Rajadhyaksha M, Goyal J, Holland C, Gorovits $\mathrm{B}$, et al. Pre-existing biotherapeutic-reactive antibodies: survey results within the American Association of Pharmaceutical Scientists. AAPS J. 2013;15(3):852-5.

30. Xue L, Rup B. Evaluation of pre-existing antibody presence as a risk factor for posttreatment anti-drug antibody induction: analysis of human clinical study data for multiple biotherapeutics. AAPS J. 2013;15(3):893-6.

31. Mire-Sluis AR, Barrett YC, Devanarayan V, Koren E, Liu H, Maia $\mathrm{M}$, et al. Recommendations for the design and optimization of immunoassays used in the detection of host antibodies against biotechnology products. J Immunol Methods. 2004;289(1-2):1-16.

32. Shankar G, Devanarayan V, Amaravadi L, Barrett YC, Bowsher R, Finco-Kent D, et al. Recommendations for the validation of immunoassays used for detection of host antibodies against biotechnology products. J Pharm Biomed Anal. 2008;48(5):1267-81.

33. USP. USP XXII-NF XVII. US Pharmacopeia. 22nd ed. Rockville; 1990. p. 644-5.

34. Mio K, Carrette O, Maibach HI, Stern R. Evidence that the serum inhibitor of hyaluronidase may be a member of the interalpha-inhibitor family. J Biol Chem. 2000;275(42):32413-21.

35. Shankar G, Arkin S, Cocea L, Devanarayan V, Kirshner S, Kromminga A, et al. Assessment and reporting of the clinical immunogenicity of therapeutic proteins and peptides-harmonized terminology and tactical recommendations. AAPS J. 2014;16(4):658-73.

36. Stern R, Jedrzejas MJ. Hyaluronidases: their genomics, structures, and mechanisms of action. Chem Rev. 2006;106(3):818-39. 
37. van der Laken CJ, Voskuyl AE, Roos JC, Stigter van Walsum $\mathrm{M}$, de Groot ER, Wolbink $\mathrm{G}$, et al. Imaging and serum analysis of immune complex formation of radiolabelled infliximab and anti-infliximab in responders and nonresponders to therapy for rheumatoid arthritis. Ann Rheum Dis. 2007;66(2):253-6.

38. Powers MR, Bell DR. Initial equilibration of albumin and IgG in rabbit hind paw skin and lymph. Microvasc Res. 1990;40(2):230-45.

39. Liadaki K, Sun J, Hammarstrom L, Pan-Hammarstrom Q. New facets of antibody deficiencies. Curr Opin Immunol. 2013;25(5):629-38.

40. Arason GJ, Jorgensen GH, Ludviksson BR. Primary immunodeficiency and autoimmunity: lessons from human diseases. Scand J Immunol. 2010;71(5):317-28.

41. Gupta S, Louis AG. Tolerance and autoimmunity in primary immunodeficiency disease: a comprehensive review. Clin Rev Allergy Immunol. 2013;45(2):162-9.

42. Baldovino S, Montin D, Martino S, Sciascia S, Menegatti E, Roccatello D. Common variable immunodeficiency: crossroads between infections, inflammation and autoimmunity. Autoimmun Rev. 2013;12(8):796-801.

43. Mital P, Hinton BT, Dufour JM. The blood-testis and bloodepididymis barriers are more than just their tight junctions. Biol Reprod. 2011;84(5):851-8.
44. Csoka AB, Scherer SW, Stern R. Expression analysis of six paralogous human hyaluronidase genes clustered on chromosomes 3p21 and 7q31. Genomics. 1999;60(3):356-61.

45. Holland MK, Andrews J, Clarke H, Walton C, Hinds LA. Selection of antigens for use in a virus-vectored immunocontraceptive vaccine: PH-20 as a case study. Reprod Fertil Dev. 1997;9(1):117-24.

46. Pomering M, Jones RC, Holland MK, Blake AE, Beagley KW. Restricted entry of IgG into male and female rabbit reproductive ducts following immunization with recombinant rabbit PH-20. Am J Reprod Immunol. 2002;47(3):174-82.

47. Hardy CM, Clydesdale G, Mobbs KJ, Pekin J, Lloyd ML, Sweet C, et al. Assessment of contraceptive vaccines based on recombinant mouse sperm protein PH20. Reproduction. 2004;127(3):325-34.

48. Primakoff P, Woolman-Gamer L, Tung KS, Myles DG. Reversible contraceptive effect of PH-20 immunization in male guinea pigs. Biol Reprod. 1997;56(5):1142-6.

49. Tung KS, Primakoff P, Woolman-Gamer L, Myles DG. Mechanism of infertility in male guinea pigs immunized with sperm PH-20. Biol Reprod. 1997;56(5):1133-41.

50. Baba D, Kashiwabara S, Honda A, Yamagata K, Wu Q, Ikawa $\mathrm{M}$, et al. Mouse sperm lacking cell surface hyaluronidase PH-20 can pass through the layer of cumulus cells and fertilize the egg. J Biol Chem. 2002;277(33):30310-4. 